TRANSACTIONS OF THE

AMERICAN MATHEMATICAL SOCIETY

Volume 353, Number 6, Pages 2275-2291

S 0002-9947(01)02780-5

Article electronically published on February 7, 2001

\title{
NONRADIAL HÖRMANDER ALGEBRAS OF SEVERAL VARIABLES AND CONVOLUTION OPERATORS
}

\author{
JOSÉ BONET, ANTONIO GALBIS, AND SIEGFRIED MOMM
}

To our friend Jean Schmets on the occasion of his 60th birthday

\begin{abstract}
A characterization of the closed principal ideals in nonradial Hörmander algebras of holomorphic functions of several variables in terms of the behaviour of the generator is obtained. This result is applied to study the range of convolution operators and ultradifferential operators on spaces of quasianalytic functions of Beurling type. Contrary to what is known to happen in the case of non-quasianalytic functions, an ultradistribution on a space of quasianalytic functions is constructed such that the range of the operator does not contain the real analytic functions.
\end{abstract}

Let $u, v: \mathbb{R} \rightarrow \mathbb{R}$ be continuous, non-negative and even functions which are increasing on the positive real numbers. We assume that $v$ is convex and the quotient $\frac{u(x)}{v(x)}$ tends to zero as $x \rightarrow \infty$. Both functions are extended to $\mathbb{R}^{N}$ as follows:

$$
u\left(x_{1}, \ldots, x_{N}\right):=\sum_{i=1}^{N} u\left(x_{i}\right), \quad v\left(x_{1}, \ldots, x_{N}\right):=\sum_{i=1}^{N} v\left(x_{i}\right) .
$$

Now we put $p(z):=u(\operatorname{Re} z)+v(\operatorname{Im} z), z \in \mathbb{C}^{N}$, and we suppose that

$$
\log (1+|z|)=O(p(z)) \text { and } p(2 z)=O(p(z)) \text { as }|z| \rightarrow \infty .
$$

Then $p$ is called a non-radial weight. The associated Hörmander algebra $A_{p}$ is the collection of all entire functions $f \in H\left(\mathbb{C}^{N}\right)$ such that, for some $k \in \mathbb{N}$,

$$
|f|_{k}:=\sup _{z \in \mathbb{C}^{N}}|f(z)| \exp (-k p(z))<\infty .
$$

In the radial case, which occurs if instead of the condition $u=o(v)$ we take $u=v$, it is well-known that each principal ideal in $A_{p}$ is closed ([2], [13]). However this is not true in general in the non-radial case. A characterization of the closed principal ideals was given by Ehrenpreis [7, 2.2] in the case $p(z)=\log \left(1+|z|^{2}\right)+|\operatorname{Im} z|$ and by Meise,Taylor and Vogt [18] in the case $v(t)=|t|$ under some particular assumptions on $u$. For general non-radial Hörmander algebras the closed principal ideals were characterized by Momm [23] in the case $N=1$. Our proposition 2 is an extension of the results in [23] to the case of functions of several variables. As a consequence,

Received by the editors June 1, 1998.

2000 Mathematics Subject Classification. Primary 46E10, 46F05, 46F10, 35R50, 32A15.

Key words and phrases. Hörmander algebras, principal ideal, convolution operators, spaces of quasianalytic functions, real analytic functions.

The research of J.Bonet and A.Galbis was supported in part by DGESIC, Proyecto no. PB970333.

(C)2001 American Mathematical Society 
some of the results in [4, [5] and [18] concerning the range of convolution operators acting on non-quasianalytic classes of Beurling type are extended to the quasianalytic case in theorem 3 and corollaries 6 and 7 . We characterize also in proposition 14 the Roumieu classes which are contained in the range of a given convolution operator, acting on a Beurling class, in terms of the behaviour of the symbol of the operator. Special emphasis is put on the case of ultradifferential operators. See propositions 9 and 15. The limit case which appears when we replace the Roumieu space by the space of the real analytic functions is considered at the end of the paper. Ehrenpreis proved in [7. Theorem II] that the intersection of the ranges of all the convolution operators on $\mathcal{E}\left(\mathbb{R}^{N}\right)$ is the space of real analytic maps $A\left(\mathbb{R}^{N}\right)$. In particular the real analytic functions are contained in the range of every convolution operator on $\mathcal{E}\left(\mathbb{R}^{N}\right)$. This result was extended by Hörmander for convex open sets (cf. [11, 16.4.5]) and for spaces of non-quasianalytic functions of Beurling type in [4. However our theorem 21 shows the existence of a (non-zero) convolution operator on a quasianalytic class of Beurling type whose range does not contain the space of the real analytic functions. This result is related to the work of Sigurdsson 30. concerning the existence of entire functions $f$ such that the limit set of $\log |f|$ is equal to a prescribed set of subharmonic functions.

Our notation is standard. We refer the reader to the books 9 , 10, 11, 15, 20,.

Remark. For $|y|$ large enough we have, for some constant $C>0$ and for all $y \in \mathbb{R}^{N}$,

$$
\begin{aligned}
v(|y|) & \leq v\left(\sum_{i=1}^{N}\left|y_{i}\right|\right) \leq \max v\left(N\left|y_{i}\right|\right) \\
& \leq C \max v\left(\left|y_{i}\right|\right) \leq C v(y) .
\end{aligned}
$$

Lemma 1. Let $v$ be an increasing and convex function on $\mathbb{R}_{+}$such that $v(2 t) \leq$ $K v(t)$ for all $t \geq 1$. Given sequences $\left(x_{j}\right) \subset \mathbb{R}^{N}$ and $\left(R_{j}\right) \subset \mathbb{R}_{+}$, there exist a positive constant $D>0$ and a sequence $\left(f_{j}\right)$ of entire functions with the following properties:

1. $\left|f_{j}(z)\right| \leq D \exp \left(D v(|\operatorname{Im} z|)+C_{N} \log \left(1+|z|^{2}\right)\right)$ if $z \in \mathbb{C}^{N},\left|z-x_{j}\right| \geq R_{j}+2$,

2. $\left|f_{j}(z)\right| \leq D \exp \left(D v\left(R_{j}\right)+C_{N} \log \left(1+|z|^{2}\right)\right)$ if $z \in \mathbb{C}^{N},\left|z-x_{j}\right| \leq R_{j}+2$,

3. $\left|f_{j}\left(x_{j}\right)\right| \geq \exp \left(v\left(\frac{2 R_{j}}{\pi \sqrt{N}}\right)-C_{N} \log \left(1+\left|x_{j}\right|^{2}\right)\right)$,

where $C_{N}$ is a positive constant which only depends on $N$.

Proof. For each $j \in \mathbb{N}$ and $R>0$ we consider the function $h_{j, R}: \mathbb{C}^{N} \longrightarrow \mathbb{R}$, which equals $|\operatorname{Im} z|$ if $\left|z-x_{j}\right| \geq R$ and with

$$
\begin{aligned}
& h_{j, R}(z):=\sup \{u(z): u \text { is plurisubharmonic on } B\left(x_{j}, R\right), \\
&\left.\qquad \varlimsup_{w \rightarrow \xi} u(w) \leq|\operatorname{Im} \xi| \text { for every } \xi \text { with }\left|\xi-x_{j}\right|=R\right\}
\end{aligned}
$$

for $\left|z-x_{j}\right|<R$. By 25], $h_{j, R}$ is plurisubharmonic and continuous on $\mathbb{C}^{N}$ and satisfies $h_{j, R}\left(x_{j}\right) \geq \frac{2 R}{\pi \sqrt{N}}$. Now we put $h_{j}:=h_{j, R_{j}}$ and define $\varphi_{j}$ as the function $\varphi_{j}(z):=h_{j, R_{j}+1}(z)+1$. Since $v$ is an increasing and convex function on $\mathbb{R}_{+}$we have that $\Phi_{j}:=v \circ \varphi_{j}$ is a plurisubharmonic function which has the following properties (see [4, p.131-132]):

(i) $\sup _{|w-z| \leq 1} \Phi_{j}(w) \leq v(|\operatorname{Im} \xi|+2)$ for every $z \in \mathbb{C}^{N},\left|z-x_{j}\right| \geq R_{j}+2$,

(ii) $\sup _{|w-z| \leq 1} \Phi_{j}(w) \leq v\left(R_{j}+4\right)$ for every $z \in \mathbb{C}^{N},\left|z-x_{j}\right|<R_{j}+2$ and 
(iii) $\inf _{\left|w-x_{j}\right| \leq 1} \Phi_{j}(w) \geq v\left(\frac{2 R_{j}}{\pi \sqrt{N}}\right)$.

Finally we apply $[9,4.4 .4]$ as in $[24,1.8]$ to find a sequence $\left(f_{j}\right)$ of entire functions with

$$
\left|f_{j}\left(x_{j}\right)\right| \geq \exp \left(\inf _{\left|w-x_{j}\right| \leq 1} \Phi_{j}(w)-C_{N} \log \left(1+\left|x_{j}\right|^{2}\right)\right)
$$

and

$$
\left|f_{j}(z)\right| \leq C_{N} \exp \left(\sup _{|z-w| \leq 1} \Phi_{j}(w)+C_{N} \log \left(1+|z|^{2}\right)\right), \quad z \in \mathbb{C}^{N},
$$

from where the conclusion follows.

For a non-radial weight $p$ we put $R_{k}(x):=v^{-1}(k u(x))$. We may assume $v(0)=0$. Then by the convexity of $v$ and the properties of $p$, for each $k \in \mathbb{N}$ there exists $n \in \mathbb{N}$ and $x_{0}>0$ with $R_{k}(x) \leq k R_{1}(x) \leq R_{n}(x), x \geq x_{0}$. Hence, in the next proposition we may replace $R_{k}$ by $k R_{1}$.

Proposition 2. Let $F \in A_{p} \backslash(0)$ be given. Then the following assertions are equivalent:

(a) There are $k \in \mathbb{N}$ and $x_{0}>0$ such that for each $x \in \mathbb{R}^{N}$ with $|x| \geq x_{0}$ there is $w \in \mathbb{C}^{N}$ with $|w-x| \leq R_{k}(x)$ and $|F(w)| \geq \exp (-k p(w))$.

(b) There are $k \in \mathbb{N}$ and $x_{0}>0$ such that for each $x \in \mathbb{R}^{N}$ with $|x| \geq x_{0}$ there is $t \in \mathbb{R}^{N}$ with $|t-x| \leq R_{k}(x)$ and $|F(t)| \geq \exp (-k u(t))$.

(c) There is $k \in \mathbb{N}$ such that for each $n \in \mathbb{N}$ there are $m \in \mathbb{N}$ and $R>0$ such that for each $z \in \mathbb{C}^{N},|z| \geq R$, there exists $w \in \mathbb{C}^{N}$ with $|w-z| \leq R_{k}(\operatorname{Re} z)+\frac{|\operatorname{Im} z|}{n}$ and $|F(w)| \geq \exp (-m p(w))$.

(d) For each $k \in \mathbb{N}$ there exist $n \in \mathbb{N}$ and $C>0$ such that for each $g \in A_{p}$ with $f:=\frac{g}{F} \in A\left(\mathbb{C}^{N}\right)$ we have $f \in A_{p}$ and $|f|_{n} \leq C|g|_{k}$.

Proof. (a) $\Rightarrow$ (b). Let $k, x_{0}$, be given by condition (a) and fix $x \in \mathbb{R}^{N}$ with $|x| \geq$ $x_{0}$. We take $w \in \mathbb{C}^{N}$ such that $|w-x| \leq R_{k}(x)$ and $|F(w)| \geq \exp (-k p(w))$ and consider the entire function $f(\xi):=F\left(\xi, w_{2}, \ldots, w_{N}\right), \xi \in \mathbb{C}$. By Levin [15] Theorem 11], applied with $\eta=\frac{3}{16}$ and $H=2+\log \left(\frac{3 e}{2 \eta}\right)$, there exists $\rho_{1}>0$ with

$$
\left|w_{1}-x_{1}\right|<\rho_{1}<\frac{3}{2}\left|w_{1}-x_{1}\right|=: R
$$

such that

$$
|f(\xi)| \geq\left|f\left(w_{1}\right)\right|^{H+1}\left(\sup _{\left|\xi-w_{1}\right|=2 e R}|f(\xi)|^{-H}\right), \quad\left|\xi-w_{1}\right|=\rho_{1} .
$$

Using that $v\left(\left|w_{1}-x_{1}\right|\right) \leq v\left(R_{k}(x)\right) \leq k u(x)$ we get the existence of constants $C_{i}$ depending on $p$ and $k$ such that

$$
\begin{aligned}
\sup _{\left|\xi-w_{1}\right|=2 e R} p(\xi) & \leq u\left(\operatorname{Re} w_{1}+2 e R\right)+v\left(\operatorname{Im} w_{1}+2 e R\right) \\
& \leq C_{1}\left(p(w)+v\left(\left|w_{1}-x_{1}\right|\right)+1\right) \\
& \leq C_{2}(p(w)+u(x)+1) .
\end{aligned}
$$

Now we choose $t_{1} \in \mathbb{R}$ with $\left|t_{1}-w_{1}\right|=\rho_{1}$. Then $\left|t_{1}-x_{1}\right| \leq \frac{5}{2} R_{k}(x)$ and $\left|F\left(t_{1}, w_{2}, \ldots, w_{N}\right)\right| \geq \exp \left(-k_{1}(p(w)+u(x)+1)\right)$ for some constant $k_{1}>0$. We again apply Levin [15, Theorem 11] to $f(\xi):=F\left(t_{1}, \xi, w_{3}, \ldots, w_{N}\right)$ to find $\rho_{2}>0$ with $\left|w_{2}-x_{2}\right|<\rho_{2}<\frac{3}{2}\left|w_{2}-x_{2}\right|,\left|t_{2}-w_{2}\right|=\rho_{2}$ and $\left|F\left(t_{1}, t_{2}, w_{3}, \ldots, w_{N}\right)\right| \geq$ 
$\exp \left(-k_{2}\left(p(w)+u(x)+u\left(t_{1}\right)+1\right)\right)$ for some constant $k_{2}>0$. Proceeding by induction in this way we obtain $t \in \mathbb{R}^{N}$ such that $\left|t_{j}-x_{j}\right| \leq \frac{5}{2} R_{k}(x)$ and

$$
|F(t)| \geq \exp \left(-k_{N}(p(w)+u(x)+u(t)+1)\right) .
$$

To finish we observe that there is $C>0$ such that $|x-t| \leq C R_{k}(x)$ and $|\omega-t| \leq$ $C R_{k}(x)$, from where it easily follows that $|F(t)| \geq \exp (-A u(t))$ for some constant $A>0$.

(b) $\Rightarrow$ (c). We modify the proof given in [23. Let $k, x_{0}$ satisfy condition (b). We can assume that $|F(z)| \leq C \exp (k p(z))$ for some constant $C>0$ and every $z \in \mathbb{C}^{N}$. We choose $k_{1} \geq k$ with $\sqrt{2} R_{k} \leq R_{k_{1}}+C_{1}$ for some $C_{1}>0$. Let $n \in \mathbb{N}$ and fix $z \in \mathbb{C}^{N}, z=x+i y, x, y \in \mathbb{R}^{N}$. We may assume that $|x| \geq x_{0}$. Otherwise we choose $w$ with respect to $z_{0}:=x_{0}+i y$ instead of $\mathrm{z}$. Condition (b) implies that there is $t \in \mathbb{R}^{N}$ such that $|t-x| \leq R_{k}(x)$ and $|F(t)| \geq \exp (-k u(t))$. We now consider two cases:

CASE I: $|y| \leq R_{k}(x)$. We put $w:=t$. Then $|w-z| \leq \sqrt{2} R_{k}(x) \leq R_{k_{1}}(x)+C_{1}$.

CASE II: $|y| \geq R_{k}(x)$. We consider the entire function $f(\xi):=F\left(\xi, t_{2}, \ldots, t_{N}\right)$, $\xi \in \mathbb{C}$. By Levin [15, Theorem 11] with $\eta=\frac{1-\left(1+\frac{1}{\sqrt{2 N n}}\right)^{-1}}{16}$ there exists $\rho_{1}>0$ with

$$
\text { (1) }\left|t_{1}-z_{1}\right|<\rho_{1}<\left(1+\frac{1}{\sqrt{2 N} n}\right)\left|t_{1}-z_{1}\right|=: R
$$

such that

$$
\text { (2) }|f(\xi)| \geq\left|f\left(t_{1}\right)\right|^{H+1}\left(\sup _{\left|\xi-t_{1}\right|=2 e R}|f(\xi)|^{-H}\right), \quad\left|\xi-t_{1}\right|=\rho_{1},
$$

with $H=2+\log \left(\frac{3 e}{2 \eta}\right)$. We choose $w_{1} \in \mathbb{C}$ with $\left|\xi-t_{1}\right|=\rho_{1}$ and

$$
\text { (3) }\left|w_{1}-z_{1}\right|<\frac{\left|t_{1}-z_{1}\right|}{\sqrt{2 N} n} \leq \frac{|y|}{\sqrt{2 N} n} .
$$

For $\xi \in \mathbb{C},\left|\xi-t_{1}\right| \leq 2 e R$ we obtain from (1),(3), with suitable constants $C_{i}>0$ depending on $p$ and $k$, that

$$
\begin{aligned}
p(\xi) & \leq C_{2} u\left(t_{1}\right)+C_{2} v\left(\left|t_{1}-z_{1}\right|\right)+C_{2} \\
& \leq C_{2} u\left(\left|x_{1}\right|+\left|t_{1}-x_{1}\right|\right)+C_{2} v(\sqrt{2}|y|)+C_{2} \\
& \leq C_{3} u(x)+C_{3} u(|t-x|)+C_{3} v(|y|)+C_{3} \\
& =C_{4} p(z)+C_{4} .
\end{aligned}
$$

Hence (since $\left|w_{1}-t_{1}\right|=\rho_{1}$ ) we get by (b) and (2) that

$$
\left|F\left(w_{1}, t_{2}, \ldots, t_{N}\right)\right| \geq \exp \left(-k^{\prime}(p(t)+p(z))-k^{\prime}\right)
$$

for some $k^{\prime}$ which depends on $p$ and $k$.

Now we again apply Levin [15, Theorem 11] in the same way to the function $f(\xi):=F\left(w_{1}, \xi, t_{3}, \ldots, t_{N}\right), \xi \in \mathbb{C}$, and obtain some $\rho_{2}>0$ with

$$
\left|t_{1}-z_{1}\right|<\rho_{1}<\left(1+\frac{1}{\sqrt{2 N} n}\right)\left|t_{1}-z_{1}\right|=: R
$$

and

$$
|f(\xi)| \geq\left|f\left(t_{2}\right)\right|^{H+1}\left(\sup _{\left|\xi-t_{2}\right|=2 e R}|f(\xi)|^{-H}\right), \quad\left|\xi-t_{2}\right|=\rho_{2} .
$$


We choose $w_{2} \in \mathbb{C}$ with $\left|w_{2}-t_{2}\right|=\rho_{2}$ and

$$
\left|w_{2}-z_{2}\right|<\frac{\left|t_{2}-z_{2}\right|}{\sqrt{2 N} n} \leq \frac{|y|}{\sqrt{2 N} n}
$$

As above we get

$$
\left|F\left(w_{1}, w_{2}, t_{3}, \ldots, t_{N}\right)\right| \geq \exp \left(-k^{\prime \prime}(p(t)+p(z))-k^{\prime \prime}\right)
$$

for some $k^{\prime \prime}$ which depends on $p$ and $k$.

Hence, by induction, we find $w_{j} \in \mathbb{C}$ with $\left|w_{j}-z_{j}\right| \leq \frac{|y|}{\sqrt{2 N} n}$ and $|F(w)| \geq$ $\exp \left(-k^{(N)}(p(t)+p(z))-k^{(N)}\right)$, where $k^{(N)}$ depends on $p, k$ and N. In particular $|w-z| \leq \frac{|y|}{n}$. We finally observe that $p(t)+p(z) \leq C_{5} p(z)+C_{5}$ for some $C_{5}>0$. Moreover $|y| \leq|\operatorname{Im} w|+\frac{|y|}{n}$, hence $|y| \leq\left(1-\frac{1}{n}\right)^{-1}|\operatorname{Im} w|$ from where it follows that $p(z)=u(x)+v(y) \leq C_{6} v(\operatorname{Im} w)+C_{6}$ for some $C_{6}>0$. Then the desired bounds for $|w-z|$ and $|F(w)|$ are proved. The additive constants which occur can be eliminated applying our result with $n+1$ instead of $n$. Then the desired results holds for large $|z| \geq R$.

$(\mathrm{c}) \Rightarrow(\mathrm{d})$. Let $k \in \mathbb{N}$ and $g \in A_{p}$ with $f:=\frac{g}{F} \in A\left(\mathbb{C}^{N}\right)$ and assume that $|g|_{k}:=\sup _{z \in \mathbb{C}^{N}}|g(z)| \exp (-k p(z))<\infty$. We take $L \in \mathbb{N}$ such that $|F|_{L}<\infty$. Given $z \in \mathbb{C}^{N}$ we apply [8, 3.2] with $r:=R_{k}(\operatorname{Re} z)+|\operatorname{Im} z|$ to get

$$
|f(z)| \leq \frac{\left(\sup _{|w-z|<4 r}|g(w)|\right)\left(\sup _{|w-z|<4 r}|F(w)|\right)}{\left(\sup _{|w-z|<r}|F(w)|\right)^{2}} .
$$

For every $|w-z| \leq 4 r$ we have $p(w) \leq u(|\operatorname{Re} z|+|w-z|)+v(|\operatorname{Im} z|+|w-z|)$. Since $u(t)=o(v(t)), p(2 z)=O(p(z))$ and $v\left(R_{k}(\operatorname{Re} z)\right)=k u(\operatorname{Re} z)$, we get $p(w) \leq$ $A(p(z)+1)$ for some constant $A>0$.

On the other hand, condition (c) with $n=1$ implies that there is $w_{0} \in \mathbb{C}^{N}$ such that $\left|w_{0}-z\right| \leq r$ and $\left|F\left(w_{0}\right)\right| \geq \exp \left(-m p\left(w_{0}\right)\right)$. Consequently

$$
|f(z)| \leq|g|_{k}|F|_{L} \exp (n p(z))
$$

for some constant $n \in \mathbb{N}$. In particular $f \in A_{p}$.

(d) $\Rightarrow$ (a). Since for every $C>0$ and $k \in \mathbb{N}$ there is $n_{0} \in \mathbb{N}$ with $C R_{k}(x) \leq R_{n}(x)$ for $n \geq n_{0}$, we have to show the following condition $(*)$ : There are $k \in \mathbb{N}, C>0$ and $x_{0}>0$ such that for each $x \in \mathbb{R}^{N}$ with $|x| \geq x_{0}$ there is $w \in \mathbb{C}^{N}$ with $|w-x| \leq C R_{k}(x)$ and $|F(w)| \geq \exp (-C k p(w))$.

Assuming that condition $(*)$ is not satisfied we can find a sequence $\left(x_{j}\right)$ in $\mathbb{R}^{N}$ with $\lim _{j \rightarrow \infty}\left|x_{j}\right|=\infty$ and such that for each $j \in \mathbb{N}$ and each $\xi \in \mathbb{C}^{N}$ with $\left|\xi-x_{j}\right| \leq C R_{j}\left(x_{j}\right)$ we have $|F(\xi)| \leq \exp (-C j p(\xi))$, where $C$ is a positive constant to be determined later. Since $u(x)=o(v(x))$ as $x$ goes to infinity we can assume $u\left(x_{j}\right) \leq \frac{1}{j} v\left(\frac{\left|x_{j}\right|}{2}\right)$, hence $R_{j}\left(x_{j}\right)=v^{-1}\left(j u\left(x_{j}\right)\right) \leq \frac{\left|x_{j}\right|}{2}$.

Let $\left(f_{j}\right)$ be a sequence of entire functions on $\mathbb{C}^{N}$ satisfying the properties (a), (b) and (c) of lemma 1 with $R_{j}:=R_{j}\left(x_{j}\right)$. It follows from property (b) that $f_{j} \in A_{p}$. In order to get a contradiction we show that $\left(F f_{j}\right)$ is bounded while $\left(f_{j}\right)$ is unbounded in $A_{p}$.

To do this we first observe that, since $\left|x_{j}\right| \leq|\operatorname{Re} \xi|+R_{j}+2 \leq|\operatorname{Re} \xi|+2+\frac{\left|x_{j}\right|}{2}$, we have $\frac{\left|x_{j}\right|}{2} \leq|\operatorname{Re} \xi|+2$. Hence there are $j_{0} \in \mathbb{N}, k>0$ and $D_{N}>0$ such that

$$
\left|f_{j}(\xi)\right| \leq D_{N} \exp \left(k j u(\operatorname{Re} \xi)+C_{N} \log \left(1+|\xi|^{2}\right)\right)
$$


for every $j \geq j_{0}$ and $\xi \in \mathbb{C}^{N},\left|\xi-x_{j}\right|<R_{j}+2$. Then, $\left|\xi-x_{j}\right|<R_{j}+2\left(\leq C R_{j}\left(x_{j}\right)\right)$ implies

$$
\left|F(\xi) f_{j}(\xi)\right| \leq D_{N} \exp \left(C_{N} \log \left(1+|\xi|^{2}\right)\right)
$$

if we take $C$ large enough at the beginning of the proof. Moreover

$$
\left|F(\xi) f_{j}(\xi)\right| \leq|F(\xi)| D_{1} \exp \left(D_{1} p(\xi)\right)
$$

for $\left|\xi-x_{j}\right| \geq R_{j}+2$. Hence there is $L \in \mathbb{N}$ with

$$
\sup _{j \in \mathbb{N}} \sup _{\xi \in \mathbb{C}^{N}}\left|F(\xi) f_{j}(\xi)\right| \exp (-L p(\xi))<\infty,
$$

and $\left(F f_{j}\right)$ is bounded. On the other hand, there is $\epsilon>0$ with $v\left(\frac{2 R_{j}\left(x_{j}\right)}{\pi \sqrt{N}}\right) \geq$ $\epsilon v\left(R_{j}\left(x_{j}\right)\right)=\epsilon j u\left(x_{j}\right)$, which implies

$$
\left|f_{j}\left(x_{j}\right)\right| \geq \exp \left(\epsilon j u\left(x_{j}\right)-C_{N} \log \left(1+\left|x_{j}\right|^{2}\right)\right)
$$

and $\left(f_{j}\right)$ is not bounded in $A_{p}$.

In 23] $(c) \Rightarrow(d)$ was proved in the case $N=1$ via another equivalent condition, namely of $F$ being slowly decreasing in the sense of Berenstein and Taylor [3]. We apply Hörmander's lemma to prove $(c) \Rightarrow(d)$.

The next characterization of the closed principal ideals in a Hörmander algebra follows from proposition 2 using standard arguments from functional analysis as in [23].

Theorem 3. For each $F \in A_{p} \backslash(0)$ the following assertions are equivalent:

(a) The principal ideal $F A_{p}$ is closed.

(b) For every $g \in A_{p}$ such that $f:=\frac{g}{F}$ is an entire function we have $f \in A_{p}$.

(c) There are $k \in \mathbb{N}$ and $x_{0}>0$ such that for each $x \in \mathbb{R}^{N}$ with $|x| \geq x_{0}$ there is $t \in \mathbb{R}^{N}$ with $|t-x| \leq R_{k}(x)$ and $|F(t)| \geq \exp (-k p(t))$.

(d) There are $k \in \mathbb{N}$ and $x_{0}>0$ such that for each $x \in \mathbb{R}^{N}$ with $|x| \geq x_{0}$ there is $\xi \in \mathbb{C}^{N}$ with $|\xi-x| \leq R_{k}(x)$ and $|F(\xi)| \geq \exp (-k p(\xi))$.

Condition (c) in theorem 3 was introduced by Ehrenpreis [7] in the case $u=\log$ and $v(t)=t$ to characterize the surjective convolution operators on the space of infinitely differentiable functions.

A continuous increasing function $\omega:[0, \infty[\longrightarrow[0, \infty[$ is called a weight if it satisfies: $\omega(2 t)=O(\omega(t)), \log \left(1+t^{2}\right)=O(\omega(t)), \omega(t)=o(t)$ as $t$ tends to $\infty$ and $\varphi: t \rightarrow \omega\left(e^{t}\right)$ is a convex function on $\mathbb{R}$.

The Young conjugate $\varphi^{*}:[0, \infty[\longrightarrow \mathbb{R}$ of $\varphi$ is defined by

$$
\varphi^{*}(s):=\sup \{s t-\varphi(t): t \geq 0\} .
$$

The weight $\omega$ is called non-quasianalytic if $\int_{1}^{\infty} t^{-2} \omega(t) d t$ is finite. See [6].

The space of ultradifferentiable functions of Beurling type $\mathcal{E}_{(\omega)}\left(\mathbb{R}^{N}\right)$ associated to a weight $\omega$ is the Fréchet-Schwartz space of all functions $f \in C^{\infty}\left(\mathbb{R}^{N}\right)$ such that for each $K \subset \mathbb{R}^{N}$ compact and each $m \in \mathbb{N}$ we have

$$
P_{K, m}(f):=\sup _{x \in K} \sup _{\alpha \in \mathbb{N}_{0}^{N}}\left|f^{(\alpha)}(x)\right| \exp \left(-m \varphi^{*}\left(\frac{|\alpha|}{m}\right)\right)<\infty .
$$


The space of ultradifferentiable functions of Roumieu type $\mathcal{E}_{\{\omega\}}\left(\mathbb{R}^{N}\right)$ associated to a weight $\omega$ is defined as the space of all functions $f \in C^{\infty}\left(\mathbb{R}^{N}\right)$ such that for each $K \subset \mathbb{R}^{N}$ compact there is $m \in \mathbb{N}$ such that

$$
\sup _{x \in K} \sup _{\alpha \in \mathbb{N}_{0}^{N}}\left|f^{(\alpha)}(x)\right| \exp \left(-\frac{1}{m} \varphi^{*}(|m \alpha|)\right)<\infty .
$$

This space is a projective limit of (DFN)-spaces which is ultrabornological. We refer to [6], 22]. Also see [32].

For $\mu \in \mathcal{E}_{(\omega)}^{\prime}\left(\mathbb{R}^{N}\right)$ the convolution operator $T_{\mu}: \mathcal{E}_{(\omega)}\left(\mathbb{R}^{N}\right) \longrightarrow \mathcal{E}_{(\omega)}\left(\mathbb{R}^{N}\right)$ is defined by $T_{\mu}(\varphi)(x):=(\mu * \varphi)(x)=\left\langle\mu_{y}, \varphi(x-y)\right\rangle$, which is a continuous linear operator with transposed map $T_{\mu}^{t}: \mathcal{E}_{(\omega)}^{\prime}\left(\mathbb{R}^{N}\right) \longrightarrow \mathcal{E}_{(\omega)}^{\prime}\left(\mathbb{R}^{N}\right), T_{\mu}^{t}(\nu):=\check{\mu} * \nu$, where $\check{\mu}(\varphi):=\mu(\check{\varphi})$ and $\check{\varphi}(x):=\varphi(-x)$.

If $\omega$ is a weight we put $u(t):=\omega(|t|), v(t):=|t|$ and we consider the associated non-radial weight function $p(z):=\omega(|\operatorname{Re} z|)+|\operatorname{Im} z|, z \in \mathbb{C}^{N}$. In this case the corresponding function $R_{k}$ is given by $R_{k}(x)=k \omega(x)$.

It is well known (see [6] 21, 22, 27]) that the Fourier-Laplace transform $F: \mu \rightarrow \widehat{\mu}$, given by $\widehat{\mu}(z):=\left\langle\mu_{x}, e^{-i\langle x, z\rangle}\right\rangle$, is an algebra isomorphism between the convolution algebra $\left(\mathcal{E}_{(\omega)}^{\prime}\left(\mathbb{R}^{N}\right), *\right)$ and the Hörmander algebra $A_{p}$ which is even a topological isomorphism when we endow $\mathcal{E}_{(\omega)}^{\prime}\left(\mathbb{R}^{N}\right)$ with the strong topology. We will identify, via Fourier-Laplace transform, the transposed map $T_{\mu}^{t}$ with the multiplication operator $F T_{\mu}^{t} F^{-1}: A_{p} \longrightarrow A_{p}, f \rightarrow \widehat{\tilde{\mu}} f$. Sometimes we will identify $\mathcal{E}_{(\omega)}^{\prime}\left(\mathbb{R}^{N}\right)$ with $A_{p}$. A standard argument gives that $T_{\mu}: \mathcal{E}_{(\omega)}\left(\mathbb{R}^{N}\right) \longrightarrow \mathcal{E}_{(\omega)}\left(\mathbb{R}^{N}\right)$ is surjective if, and only if, the principal ideal $\widehat{\mu} A_{p}$ is closed in $A_{p}$.

We do not assume in the rest of the article that the weight $\omega$ is non-quasianalytic.

Examples 4. (a) $\omega(r)=r^{1 / t}, t>1$, is a non-quasianalytic weight. In this case $\mathcal{E}_{\{\omega\}}\left(\mathbb{R}^{N}\right)=\Gamma^{\{t\}}\left(\mathbb{R}^{N}\right)$ is the Gevrey space of order $t$.

(b) $\omega(r)=\frac{r}{\log (r+e)^{q}}, 0<q \leq 1$, is a quasianalytic weight.

Definition 5. An ultradistribution $\mu \in \mathcal{E}_{(\omega)}^{\prime}\left(\mathbb{R}^{N}\right)$ is called slowly decreasing for $(\omega)$ if there are $k \in \mathbb{N}$ and $x_{0}>0$ such that for each $x \in \mathbb{R}^{N}$ with $|x| \geq x_{0}$ there is $\xi \in \mathbb{C}^{N}$ with $|\xi-x| \leq k \omega(x)$ and $|\hat{\mu}(\xi)| \geq \exp (-k \omega(\xi)-k|\operatorname{Im} \xi|)$.

It follows from proposition 2 that the ultradistribution $\mu \in \mathcal{E}_{(\omega)}^{\prime}\left(\mathbb{R}^{N}\right)$ is slowly decreasing for $(\omega)$ if and only if there exists $k \in \mathbb{N}$ such that for each $j \in \mathbb{N}$ there exist $m \in \mathbb{N}$ and $C>0, R>0$ such that for each $z \in \mathbb{C}^{N},|z| \geq R$, there exists $w \in \mathbb{C}^{N}$ satisfying

$$
|w-z| \leq k \omega(z)+\frac{1}{j}|\operatorname{Im} z| \text { and }|\hat{\mu}(w)| \geq C \exp (-m(|\operatorname{Im} w|+\omega(w))) .
$$

The above reformulation of the slowly decreasing condition has been used in [5]. In the case $N=1$, theorem 3 implies that our definition of $(\omega)$-slowly decreasing coincides with the one used by Meise, Taylor and Vogt in [18.

As an immediate consequence of theorem 3 we get

Corollary 6. Let $\omega$ be a weight and let $\mu \in \mathcal{E}_{(\omega)}^{\prime}\left(\mathbb{R}^{N}\right)$. Then the following conditions are equivalent:

(a) $T_{\mu}: \mathcal{E}_{(\omega)}\left(\mathbb{R}^{N}\right) \rightarrow \mathcal{E}_{(\omega)}\left(\mathbb{R}^{N}\right)$ is surjective,

(b) $\mu$ is slowly decreasing for $(\omega)$. 
This result was already proved in 18 in the non-quasianalytic case and for $N=1$. A proof for arbitrary $N$ and non-quasianalytic weights can be found in [4]. The proof in [4], 18] depended on an application of the Phragmén-Lindelöf theorem which was possible because the non-quasianalyticity of the weight $\omega$ permits us to extend it to a harmonic function in the upper halfplane. The proof of the corollary for arbitrary weights depends on the equivalence between conditions (a) and (c) of proposition 2 .

The next corollary extends [4, Theorem 2] and part of [5, 2.8] to quasianalytic weights.

Corollary 7. Let $\omega \leq \sigma$ be two weights. The following conditions are equivalent for $\mu \in \mathcal{E}_{(\omega)}^{\prime}\left(\mathbb{R}^{N}\right)$ :

(a) $\mathcal{E}_{(\sigma)}\left(\mathbb{R}^{N}\right)$ is contained in $T_{\mu}\left(\mathcal{E}_{(\omega)}\left(\mathbb{R}^{N}\right)\right)$.

(b) If $B \subset \mathcal{E}_{(\omega)}^{\prime}\left(\mathbb{R}^{N}\right)$ satisfies that $T_{\mu}^{t}(B)$ is bounded in $\mathcal{E}_{(\omega)}^{\prime}\left(\mathbb{R}^{N}\right)$ then $B$ is bounded in $\mathcal{E}_{(\sigma)}^{\prime}\left(\mathbb{R}^{N}\right)$.

(c) $T_{\mu}: \mathcal{E}_{(\sigma)}\left(\mathbb{R}^{N}\right) \longrightarrow \mathcal{E}_{(\sigma)}\left(\mathbb{R}^{N}\right)$ is surjective.

Proof. To prove that (a) implies (b) we may proceed as in [4, Theorem 2], since the proof of $(1) \Rightarrow(3)$ in [4, Proposition 1] remains valid for quasianalytic weights. To prove that (b) implies (c) we first proceed as in 4, Theorem 2] to deduce that $\mu$ is slowly decreasing for $(\sigma)$ and then we apply corollary 6 .

If $G \in \mathcal{H}\left(\mathbb{C}^{N}\right)$ satisfies $\log |G|=\mathcal{O}(\omega)$ then $\mu_{G}(\varphi)=\sum_{\alpha}(-i)^{|\alpha|} \frac{G^{(\alpha)}(0)}{\alpha !} \varphi^{(\alpha)}(0)$ defines an element $\mu_{G}$ of $\mathcal{E}_{(\omega)}^{\prime}\left(\mathbb{R}^{N}\right)$ with support $\{0\}$. The operator

$$
G(D): \mathcal{E}_{(\omega)}\left(\mathbb{R}^{N}\right) \longrightarrow \mathcal{E}_{(\omega)}\left(\mathbb{R}^{N}\right), \quad G(D) f=\mu_{G} * f
$$

is then called an ultradifferential operator of class $(\omega)$. Note that $G(D) f(x)=$ $\sum_{\alpha} i^{|\alpha|} \frac{G^{(\alpha)}(0)}{\alpha !} f^{(\alpha)}(x)$.

For the convenience of the reader we state the following result which will be used in the sequel. It permits us to construct entire functions with certain growth properties and vanishing on a given distribution of points.

Theorem 8 (Rubel and Taylor 28] 29]). Let $\lambda(r), r \geq 0$, be a positive, increasing and unbounded function such that $\lambda(2 r) \leq M \lambda(r)$ for some $M>0$. Given two sequences $\left(a_{j}\right) \subset \mathbb{C} \backslash\{0\}$ and $\left(m_{j}\right) \subset \mathbb{N}$ we put $n(t):=\sum_{\left|a_{j}\right| \leq t} m_{j}$ and $N(r):=$ $\int_{0}^{r} \frac{n(t)}{t} d t$. If there are positive constants $A, B$ such that $N(r) \leq A \lambda(B r), r>0$, then there exist an entire function $f \in H(\mathbb{C}), f \neq 0$, such that the inequality $|f(z)| \leq \exp \left(A^{\prime} \lambda\left(B^{\prime}|z|\right)\right)$ holds for some constants $A^{\prime}, B^{\prime}$, and $f\left(a_{j}\right)=0$ with multiplicity at least $m_{j}(j \in \mathbb{N})$.

Our next result exploits ideas and methods of [26].

Proposition 9. Let $\omega \leq \sigma$ be two weights. Then the following conditions are equivalent:

(a) $\mathcal{E}_{(\sigma)}\left(\mathbb{R}^{N}\right)$ is contained in $G(D)\left(\mathcal{E}_{(\omega)}\left(\mathbb{R}^{N}\right)\right)$ for every ultradifferential operator $G(D)$, of class $(\omega)$.

(b) There are $C>0$ and $R_{0}>0$ such that for each $R \geq R_{0}$ we have

$$
\inf _{S>R} \frac{\omega(S)}{\log \left(\frac{S}{R}\right)} \leq C \frac{\sigma(R)}{\log \left(\frac{R}{\sigma(R)}\right)} .
$$


Proof. (a) $\Rightarrow$ (b). Proceeding by contradiction we assume

$$
\forall C>0 \quad \forall R_{0}>0 \quad \exists R \geq R_{0}: \inf _{S>R} \frac{\omega(S)}{\log \left(\frac{S}{R}\right)}>C \frac{\sigma(R)}{\log \left(\frac{R}{\sigma(R)}\right)} .
$$

Inductively we select an increasing and unbounded sequence $\left(a_{j}\right)$ of positive real numbers and a sequence $\left(m_{j}\right) \subset \mathbb{N}$ such that

(i) $\inf _{S>a_{j}} \frac{\omega(S)}{\log \left(\frac{S}{a_{j}}\right)}>m_{j}>j \frac{\sigma\left(a_{j}\right)}{\log \left(\frac{a_{j}}{\sigma\left(a_{j}\right)}\right)}$,

(ii) $n\left(a_{j-1}\right) \leq m_{j}$,

(iii) $n\left(a_{j-1}\right) \log \left(\frac{a_{j}}{a_{1}}\right) \leq \omega\left(a_{j}\right)$, where $n(t):=\sum_{a_{j} \leq t} m_{j}$. To apply the Theorem of Rubel and Taylor mentioned above, for $\lambda=\omega$ we must estimate $N(r):=\int_{0}^{r} \frac{n(t)}{t} d t$. For $a_{j}<r<a_{j+1}$ we have

$$
\begin{aligned}
N(r) & =\int_{a_{1}}^{a_{j}} \frac{n(t)}{t} d t+\int_{a_{j}}^{r} \frac{n(t)}{t} d t \\
& \leq n\left(a_{j-1}\right) \int_{a_{1}}^{a_{j}} \frac{d t}{t}+\left(n\left(a_{j-1}\right)+m_{j}\right) \int_{a_{j}}^{r} \frac{d t}{t} \\
& \leq \omega\left(a_{j}\right)+2 m_{j} \log \left(\frac{r}{a_{j}}\right) .
\end{aligned}
$$

Since $\frac{\omega(r)}{\log \left(\frac{r}{a_{j}}\right)}>m_{j}$ we conclude $N(r) \leq \omega\left(a_{j}\right)+2 \omega(r) \leq 3 \omega(r)$. Consequently there is an entire function $F \in H(\mathbb{C}), F \neq 0$, such that $F\left(a_{j}\right)=0$ with multiplicity at least $m_{j}, j \in \mathbb{N}$, and $|F(z)| \leq \exp (A \omega(|z|))$ for some $A>0$.

By corollary 7 , it is enough to show that $F$ is not slowly decreasing for $(\sigma)$ to conclude. Let $k>0$ be arbitrary, $w=a_{j}+r e^{i \theta}, 0 \leq r \leq k \sigma\left(a_{j}\right)$. Then we have, by the Jensen-Poisson formula, for $z=a_{j}, R=|z|=a_{j}$,

$$
\begin{aligned}
\log |F(w)| & =\frac{1}{2 \pi} \int_{0}^{2 \pi} P_{\frac{r}{R}}(\theta-t) \log \left|F\left(z+R e^{i t}\right)\right| d t \\
& -\sum_{|z-a| \leq R, F(a)=0} \log \left|\frac{R^{2}-\overline{(a-z)} r e^{i \theta}}{R\left(r e^{i \theta}-(a-z)\right)}\right| .
\end{aligned}
$$

Since each term in the sum is positive

$$
\begin{aligned}
\log |F(w)| & \leq \frac{1}{2 \pi} \int_{0}^{2 \pi} P_{\frac{r}{R}}(\theta-t) \log \left|F\left(z+R e^{i t}\right)\right| d t-m_{j} \log \left(\frac{R}{r}\right) \\
& \leq \sup _{\xi \in \mathbb{C} ;|\xi| \leq 2|z|} \log |F(\xi)|-m_{j} \log \left(\frac{R}{r}\right) \\
& \leq A \omega(2|z|)-m_{j} \log \left(\frac{a_{j}}{k \sigma\left(a_{j}\right)}\right)
\end{aligned}
$$

This means that $\log |F(w)| \leq A \omega(2|z|)-m_{j} \log \left(\frac{a_{j}}{k \sigma\left(a_{j}\right)}\right)$ whenever we have $\left|\omega-a_{j}\right| \leq$ $k \sigma\left(a_{j}\right)$. Now it is enough to note that

$$
A \omega(2|z|)-m_{j} \log \left(\frac{a_{j}}{k \sigma\left(a_{j}\right)}\right) \leq-k \sigma\left(a_{j}\right)
$$

for $j$ large enough in order to conclude that $G(z):=F\left(z_{1}\right), z \in \mathbb{C}^{N}$, defines an ultradifferential operator $G(D)$ of class $(\omega)$ such that $\mathcal{E}_{(\sigma)}\left(\mathbb{R}^{N}\right)$ is not contained in $G(D)\left(\mathcal{E}_{(\omega)}\left(\mathbb{R}^{N}\right)\right)$. 
(b) $\Rightarrow$ (a). We first assume $G(0) \neq 0$. Without loss of generality we can take $G(0)=1$. We fix $z \in \mathbb{C}^{N}$ with $|z| \geq R_{0}$ (as in (b)) and set $R:=|z|$. There is $S>R$ such that

$$
\frac{\omega(S)}{\log \left(\frac{S}{R}\right)} \leq C \frac{\sigma(R)}{\log \left(\frac{R}{\sigma(R)}\right)}
$$

We take $\eta:=\frac{3}{2} e \frac{\sigma(R)}{R}<\frac{3}{2} e$ and $g(\xi):=G\left(\frac{\xi z}{|z|}\right), \xi \in \mathbb{C}$. By the minimum modulus theorem of Levin [15. Theorem 11], in the more general form given by [26, 4.2], there are discs $\left(K_{j}\right)_{j=1}^{n}$ the sum of their radii is less than or equal to $4 \eta R$ and such that for every $\xi \notin \mathbb{C},|\xi|<R, \xi \in \bigcup K_{j}$ one has

$$
\log |g(\xi)| \geq-2 \log M(2 R)-\log \left(\frac{3 e}{2 \eta}\right) \frac{\log M(2 S)}{\log \left(\frac{S}{R}\right)},
$$

where $M(t)=\sup _{|z|=t}|g(z)|$. Since $8 \eta R=12 e \sigma(R)<A \sigma(R)<R$ for some $A>0$ and $R$ large enough, we conclude the existence of $\xi \in \mathbb{C}$ with $|\xi-R|<A \sigma(R)$, $|\xi| \leq R$, and

$$
|g(\xi)| \geq \exp \left(-2 \log M(2 R)-\log \left(\frac{3 e}{2 \eta}\right) \frac{\log M(2 S)}{\log \left(\frac{S}{R}\right)}\right) .
$$

Then for $w=\frac{\xi z}{|z|}$ we have $|w-z|=|\xi-R|<A \sigma(R)$ and, for some $k>0$,

$$
\begin{aligned}
\log |G(w)| & \geq-2 \log M(2 R)-\log \left(\frac{3 e}{2 \eta}\right) \frac{\log M(2 S)}{\log \left(\frac{S}{R}\right)} \\
& \geq-2 k \omega(2 R)-\log \left(\frac{R}{\sigma(R)}\right) k \frac{\omega(2 S)}{\log \left(\frac{S}{R}\right)} \\
& \geq-2 k \omega(2 R)-k C \sigma(R) \geq-B \sigma(R)
\end{aligned}
$$

for some constant $B>0$. Corollary 7 permits to conclude that $\mathcal{E}_{(\sigma)}\left(\mathbb{R}^{N}\right)$ is contained in $G(D)\left(\mathcal{E}_{(\omega)}\left(\mathbb{R}^{N}\right)\right)$. In case $G(0)=0$ we choose $w \in \mathbb{C}^{N}$ with $G(w) \neq 0$ and proceed as above with the function $G_{1}(z):=G(z+w)$.

Examples 10. (a) Let $\omega \leq \sigma$ be two non-quasianalytic weights. If $\mu \in D_{(\sigma)}\left(\mathbb{R}^{N}\right) \subset$ $\mathcal{E}_{(\omega)}^{\prime}\left(\mathbb{R}^{N}\right)$, then $\mathcal{E}_{(\sigma)}\left(\mathbb{R}^{N}\right)$ is not contained in $T_{\mu}\left(\mathcal{E}_{(\omega)}\left(\mathbb{R}^{N}\right)\right)$. In fact, it follows from the Paley-Wiener theorem ([6]) that $\mu$ is not slowly decreasing for $(\sigma)$. We then apply corollaries 6 and 7 .

(b) Let $1<s<t$ and $\omega(r)=r^{1 / t}, \sigma(r)=r^{1 / s}$. Then $\mathcal{E}_{(\sigma)}\left(\mathbb{R}^{N}\right)$ is contained in $G(D)\left(\mathcal{E}_{(\omega)}\left(\mathbb{R}^{N}\right)\right.$ ), for every ultradifferential operator $G(D)$ of class $(\omega)$. In fact, we take $S=2 R$ and observe that

$$
\frac{\omega(S)}{\log \left(\frac{S}{R}\right)} \frac{\log \left(\frac{R}{\sigma(R)}\right)}{\sigma(R)} \rightarrow 0 \quad \text { for } R \rightarrow \infty
$$

The conclusion follows from proposition 9 .

(c) Ehrenpreis [7] proved that $\mathcal{E}_{\{\tau\}}\left(\mathbb{R}^{N}\right)$ is contained in $T_{\mu}\left(\mathcal{C}^{\infty}\left(\mathbb{R}^{N}\right)\right)$ for every $\mu \in \mathcal{E}^{\prime}\left(\mathbb{R}^{N}\right)$ if $\tau(t)=|t|$.

Lemma 11. Let $\omega \leq \sigma$ be two weights and let $\mu \in \mathcal{E}_{(\omega)}^{\prime}\left(\mathbb{R}^{N}\right)$ be an ultradistribution such that the range of $T_{\mu}: \mathcal{E}_{(\omega)}\left(\mathbb{R}^{N}\right) \longrightarrow \mathcal{E}_{(\omega)}\left(\mathbb{R}^{N}\right)$ contains the Roumieu class $H:=$ 
$\mathcal{E}_{\{\sigma\}}\left(\mathbb{R}^{N}\right)$. If $B \subset \mathcal{E}_{(\omega)}^{\prime}\left(\mathbb{R}^{N}\right)$ satisfies that $T_{\mu}^{t}(B)$ is equicontinuous in $\mathcal{E}_{(\omega)}^{\prime}\left(\mathbb{R}^{N}\right)$, then $\left\{\left.\varphi\right|_{H}: \varphi \in B\right\}$ is equicontinuous in $\mathcal{E}_{\{\sigma\}}^{\prime}\left(\mathbb{R}^{N}\right)$.

Proof. Let $\tau$ denote the quotient topology induced by $T_{\mu}$ on its range. Then $G:=\left(T_{\mu}\left(\mathcal{E}_{(\omega)}\left(\mathbb{R}^{N}\right)\right), \tau\right)$ is a Fréchet space and the inclusion $i: \mathcal{E}_{\{\sigma\}}\left(\mathbb{R}^{N}\right) \rightarrow G$ is continuous, since it is a linear map with closed graph. We now fix a 0-neighbourhood $V$ in $\mathcal{E}_{(\omega)}\left(\mathbb{R}^{N}\right)$ such that $\left|\left\langle T_{\mu}^{t} \varphi, f\right\rangle\right| \leq 1$ for every $\varphi \in B$ and $f \in V$. Then $W:=T_{\mu}(V) \cap \mathcal{E}_{\{\sigma\}}\left(\mathbb{R}^{N}\right)$ is a neighbourhood in $\mathcal{E}_{\{\sigma\}}\left(\mathbb{R}^{N}\right)$ and $|\langle\varphi, g\rangle| \leq 1$ for every $g \in W$ and $\varphi \in B$.

Definition $12([5])$. An ultradistribution $\mu \in \mathcal{E}_{\{\omega\}}^{\prime}\left(\mathbb{R}^{N}\right)$ is called slowly decreasing for $\{\omega\}$ if for each $m \in \mathbb{N}$ there exists $R>0$ such that for each $x \in \mathbb{R}^{N}$ with $|x| \geq R$ there exists $\xi \in \mathbb{C}^{N}$ satisfying $|x-\xi| \leq \frac{1}{m} \omega(x)$ such that $|\hat{\mu}(\xi)| \geq \exp \left(-\frac{1}{m} \omega(\xi)\right)$.

Proceeding as in [5] 3.2] it turns out that $\mu \in \mathcal{E}_{\{\omega\}}^{\prime}\left(\mathbb{R}^{N}\right)$ is slowly decreasing for $\{\omega\}$ if, and only if, there exists a weight function $\sigma$ with $\sigma=o(\omega)$ such that $\mu \in \mathcal{E}_{(\sigma)}^{\prime}\left(\mathbb{R}^{N}\right)$ and $\mu$ is slowly decreasing for $(\sigma)$.

By [6, 22, 27], the Fourier-Laplace transform $F: \mu \rightarrow \widehat{\mu}$ defines a topological isomorphism between the (LF)-spaces $\mathcal{E}_{\{\omega\}}^{\prime}\left(\mathbb{R}^{N}\right)$ and $\operatorname{ind}_{j} \operatorname{proj}_{k} A\left(\omega_{j k}, \mathbb{C}^{N}\right)$, where

$$
A\left(\omega_{j k}, \mathbb{C}^{N}\right):=\left\{f \in H\left(\mathbb{C}^{N}\right): \sup _{z \in \mathbb{C}^{N}} \omega_{j k}(z)|f(z)|<\infty\right\},
$$

and

$$
\omega_{j k}(z):=\exp \left(-j|\operatorname{Im} z|-\frac{1}{k} \omega(z)\right) .
$$

The following lemma extends [19, 3.2,3.3] to quasianalytic classes. We indicate the similar proof. We thank A. Albanese and R. Meise for conversations on the lemma.

Lemma 13. Let $\sigma$ be a weight. For every equicontinuous set $A \subset \mathcal{E}_{\{\sigma\}}^{\prime}\left(\mathbb{R}^{N}\right)$, there is a weight $\tau=o(\sigma)$ and there is a seminorm $P$ on $\mathcal{E}_{\{\tau\}}\left(\mathbb{R}^{N}\right)$ such that $|\varphi(f)| \leq$ $P(f)$ for every $\varphi \in B$ and every $f \in \mathcal{E}_{\{\sigma\}}^{\prime}\left(\mathbb{R}^{N}\right)$.

Proof. We identify spaces via the Fourier-Laplace transform. Since $A$ is equicontinuous, it is contained in a Banach disc in $\operatorname{ind}_{j} \operatorname{proj}_{k} A\left(\omega_{j k}, \mathbb{C}^{N}\right)$. By Grothendieck's factorization theorem, it is bounded in a step, i.e.

$$
\begin{aligned}
& \exists j \forall k \exists M_{k} \forall \nu \in A \forall z \in \mathbb{C}^{N}: \\
& |\widehat{\nu}(z)| \leq M_{k} \exp \left(j|\operatorname{Im} z|+\frac{1}{k} \sigma(z)\right) .
\end{aligned}
$$

We get, for each $k$ and $r>0$

$$
g(r):=\sup _{|z|=r} \log ^{+}(|\widehat{\nu}(z)| \exp (-j|\operatorname{Im} z|)) \leq \frac{1}{k} \sigma(r)+\log M_{k},
$$

i.e., $g=o(\sigma)$. By [6] 1.7 and 1.8], there is a weight $\tau=o(\sigma)$ such that $g=o(\sigma)$. This yields

$$
\begin{gathered}
\forall \delta>0 \exists C_{\delta}>0 \forall \nu \in B \forall z \in \mathbb{C}^{N}: \\
|\widehat{\nu}(z)| \leq C_{\delta} \exp (j|\operatorname{Im} z|+\delta \tau(z)) .
\end{gathered}
$$

This implies that $A$ is bounded, hence equicontinuous in $\mathcal{E}_{\{\tau\}}^{\prime}\left(\mathbb{R}^{N}\right)$ and the proof is complete. 
Proposition 14. Let $\omega \leq \sigma$ be two weights, $\omega=o(\sigma)$ and let $\mu \in \mathcal{E}_{(\omega)}^{\prime}\left(\mathbb{R}^{N}\right)$. Then, the following conditions are equivalent:

(a) $\mathcal{E}_{\{\sigma\}}\left(\mathbb{R}^{N}\right)$ is contained in $T_{\mu}\left(\mathcal{E}_{(\omega)}\left(\mathbb{R}^{N}\right)\right)$.

(b) There is a weight $\tau$ satisfying $\omega=o(\tau)$ and $\tau=o(\sigma)$ such that $\mathcal{E}_{(\tau)}\left(\mathbb{R}^{N}\right)$ is contained in $T_{\mu}\left(\mathcal{E}_{(\omega)}\left(\mathbb{R}^{N}\right)\right)$.

(c) $\mu$ is slowly decreasing for $\{\sigma\}$.

Proof. (a) $\Rightarrow$ (b). Let $\left(C_{n}\right)$ be a fundamental sequence of bounded sets in $\mathcal{E}_{(\omega)}^{\prime}\left(\mathbb{R}^{N}\right)$. We put $G:=\mathcal{E}_{\{\sigma\}}\left(\mathbb{R}^{N}\right)$. By lemma 11 , for each $n \in \mathbb{N}$, the set $B_{n}:=\left\{\left.\varphi\right|_{G}\right.$ : $\left.T_{\mu}^{t} \varphi \in C_{n}\right\}$ is equicontinuous in $\mathcal{E}_{\{\sigma\}}^{\prime}\left(\mathbb{R}^{N}\right)$. We can apply lemma 13 to find a weight $\sigma_{n}=o(\sigma)$ and a seminorm $P_{n}$ on $\mathcal{E}_{\left(\sigma_{n}\right)}\left(\mathbb{R}^{N}\right)$ such that $|\varphi(f)| \leq P_{n}(f)$ for every $\varphi \in B_{n}$ and $f \in G$. By [6, 1.9] we find a weight $\tau$ with $\sigma_{n}=o(\tau)$ for every $n \in \mathbb{N}, \omega=o(\tau)$ and $\tau=o(\sigma)$. Clearly $\mathcal{E}_{\{\sigma\}}\left(\mathbb{R}^{N}\right) \subset \mathcal{E}_{(\tau)}\left(\mathbb{R}^{N}\right) \subset \mathcal{E}_{(\omega)}\left(\mathbb{R}^{N}\right)$. Fix $B \subset \mathcal{E}_{(\omega)}^{\prime}\left(\mathbb{R}^{N}\right)$ such that $T_{\mu}^{t}(B)$ is a bounded set in $\mathcal{E}_{(\omega)}^{\prime}\left(\mathbb{R}^{N}\right)$. There is $m \in \mathbb{N}$ with $T_{\mu}^{t}(B) \subset C_{m}$. Accordingly $B$ is bounded in $\mathcal{E}_{\left(\sigma_{m}\right)}^{\prime}\left(\mathbb{R}^{N}\right)$, hence in $\mathcal{E}_{(\tau)}^{\prime}\left(\mathbb{R}^{N}\right)$. We apply corollary 7 to conclude that $\mathcal{E}_{(\tau)}\left(\mathbb{R}^{N}\right)$ is contained in $T_{\mu}\left(\mathcal{E}_{(\omega)}\left(\mathbb{R}^{N}\right)\right)$.

(b) $\Rightarrow$ (c). Since $\tau=o(\sigma)$, we can apply corollaries 6 and 7 to conclude that $\mu$ is slowly decreasing for $(\tau)$.

(c) $\Rightarrow$ (a) follows from the inclusion $\mathcal{E}_{\{\sigma\}}\left(\mathbb{R}^{N}\right) \subset \mathcal{E}_{(\tau)}\left(\mathbb{R}^{N}\right)$.

Proposition 15. Let $\omega \leq \sigma$ be two weights such that $\omega=o(\sigma)$. The following conditions are equivalent:

(a) $\mathcal{E}_{\{\sigma\}}\left(\mathbb{R}^{N}\right)$ is contained in $G(D)\left(\mathcal{E}_{(\omega)}\left(\mathbb{R}^{N}\right)\right)$ for every ultradifferential operator $G(D)$ of class $(\omega)$.

(b) For every $\epsilon>0$ there is $R_{0}>0$ such that for each $R \geq R_{0}$ we have

$$
\inf _{S>R} \frac{\omega(S)}{\log \left(\frac{S}{R}\right)} \leq \epsilon \frac{\sigma(R)}{\log \left(\frac{R}{\epsilon \sigma(R)}\right)} .
$$

(c) There is a weight function $\tau, \omega \leq \tau, \tau=o(\sigma)$, such that $\mathcal{E}_{(\tau)}\left(\mathbb{R}^{N}\right)$ is contained in $G(D)\left(\mathcal{E}_{(\omega)}\left(\mathbb{R}^{N}\right)\right)$ for every ultradifferential operator $G(D)$ of class $(\omega)$.

Proof. (a) $\Rightarrow$ (b). Proceeding by contradiction we assume

$$
\exists 0<\epsilon<\frac{1}{3} \quad \forall R_{0}>0 \quad \exists R \geq R_{0}: \inf _{S>R} \frac{\omega(S)}{\log \left(\frac{S}{R}\right)}>\epsilon \frac{\sigma(R)}{\log \left(\frac{R}{\epsilon \sigma(R)}\right)} .
$$

Inductively we select an increasing and unbounded sequence $\left(a_{j}\right)$ of positive real numbers and a sequence $\left(m_{j}\right) \subset \mathbb{N}$ such that

(i) $\inf _{S>a_{j}} \frac{\omega(S)}{\log \left(\frac{S}{a_{j}}\right)}>m_{j}>3 \epsilon \frac{\sigma\left(a_{j}\right)}{\log \left(\frac{a_{j}}{\epsilon \sigma\left(a_{j}\right)}\right)}$,

(ii) $n\left(a_{j-1}\right) \leq m_{j}$,

(iii) $n\left(a_{j-1}\right) \log \left(\frac{a_{j}}{a_{1}}\right) \leq \omega\left(a_{j}\right)$, where $n(t):=\sum_{a_{j} \leq t} m_{j}$. To apply the Theorem of

Rubel and Taylor for $\lambda=\omega$ we must estimate $N(r):=\int_{0}^{r} \frac{n(t)}{t} d t$. For $a_{j}<r<a_{j+1}$ we have (as in the proof of proposition 9)

$$
N(r) \leq \omega\left(a_{j}\right)+2 m_{j} \log \left(\frac{r}{a_{j}}\right) .
$$

Since $\frac{\omega(r)}{\log \left(\frac{r}{a_{j}}\right)}>\epsilon m_{j}$ we conclude $N(r) \leq\left(1+\frac{2}{\epsilon}\right) \omega(r)$. Consequently there is an entire function $F \in H(\mathbb{C}), F \neq 0$, such that $F\left(a_{j}\right)=0$ with multiplicity at least $m_{j}, j \in \mathbb{N}$, and $|F(z)| \leq \exp (A \omega(|z|))$ for some $A>0$. To conclude, it is enough 
to show that $F$ is not slowly decreasing for $\{\sigma\}$. Let $w=a_{j}+r e^{i \theta}, 0 \leq r \leq \epsilon \sigma\left(a_{j}\right)$. Then we have, by the Jensen-Poisson formula, for $z=a_{j}, R=|z|=a_{j}$,

$$
\log |F(w)| \leq A \omega(2|z|)-m_{j} \log \left(\frac{a_{j}}{\epsilon \sigma\left(a_{j}\right)}\right) .
$$

This means that $\log |F(w)| \leq A \omega\left(2 a_{j}\right)-m_{j} \log \left(\frac{a_{j}}{\epsilon \sigma\left(a_{j}\right)}\right)$ whenever $\left|\omega-a_{j}\right| \leq \epsilon \sigma\left(a_{j}\right)$. Now it is enough to note that

$$
A \omega\left(2 a_{j}\right)-m_{j} \log \left(\frac{a_{j}}{\epsilon \sigma\left(a_{j}\right)}\right) \leq-\epsilon \sigma\left(a_{j}\right)
$$

for $j$ large enough to conclude that $G(z):=F\left(z_{1}\right), z \in \mathbb{C}^{N}$, defines an ultradifferential operator $G(D)$ of class $(\omega)$ such that $\mathcal{E}_{\{\sigma\}}\left(\mathbb{R}^{N}\right)$ is not contained in $G(D)\left(\mathcal{E}_{(\omega)}\left(\mathbb{R}^{N}\right)\right)$.

(b) $\Rightarrow$ (c). We find an increasing sequence $\left(R_{m}\right)$ of positive real numbers such that for every $r \geq R_{m}$ :

$$
\inf _{S>R} \frac{\omega(S)}{\log \left(\frac{S}{R}\right)} \leq \frac{1}{m} \frac{\sigma(R)}{\log \left(\frac{m R}{\sigma(R)}\right)}
$$

We define $g(t)=0$ for $t \in\left[0, R_{1}\left[\right.\right.$ and $g(t)=\frac{\sigma(x)}{m}$ for $t \in\left[R_{m}, R_{m+1}\right.$ [ and apply [6, 1.7;1.8] to find a weight function $\tau$ such that $\omega \leq \tau, g=o(\tau), \tau=o(\sigma)$. We apply proposition 9 to get that $\mathcal{E}_{(\tau)}\left(\mathbb{R}^{N}\right)$ is contained in $G(D)\left(\mathcal{E}_{(\omega)}\left(\mathbb{R}^{N}\right)\right)$ for every ultradifferential operator $G(D)$ of class $(\omega)$.

Example 16. Let $\omega(r)=o\left(\frac{r^{1 / t}}{\log r}\right), t>1$, and $\sigma(r)=r^{1 / t}$. Then $\mathcal{E}_{\{\sigma\}}\left(\mathbb{R}^{N}\right)$ is contained in $G(D)\left(\mathcal{E}_{(\omega)}\left(\mathbb{R}^{N}\right)\right.$ ), for every ultradifferential operator $G(D)$ of class $(\omega)$.

In fact, we put $\Phi(R):=\inf _{S>R} \frac{\omega(S)}{\log \left(\frac{S}{R}\right)}$. Using that $\Phi(R) \leq \frac{\omega(2 R)}{\log 2}$ we deduce that $\Phi(R)=o\left(\frac{\sigma(R)}{\log R}\right)$. Consequently, for every $0<\epsilon<1$ there is $R_{1}>0$ such that $R \geq R_{1}$ implies $\Phi(R) \leq \epsilon \frac{\sigma(R)}{\log R}$. Hence $\Phi(r) \frac{\log \left(\frac{R}{\epsilon \sigma(R)}\right)}{\sigma(R)} \leq \epsilon \frac{\log \left(\frac{R}{\epsilon \sigma(R)}\right)}{\log R}$. Since the term in the right-hand side converges to $\left(1-\frac{1}{t}\right) \epsilon$ as $R \rightarrow \infty$ it follows that condition (b) in the proposition 15 is satisfied.

The example above is "sharp" since for a weight $\omega$ with $2 \omega(r) \leq \omega(A r), r \geq r_{0}$, for some $A>1$ we have $\inf _{S>R} \frac{\omega(S)}{\log \left(\frac{S}{R}\right)} \sim \omega(R)$ by [24, ex.4.6(b)].

Remark. The condition (b) in proposition 15 is equivalent to the following:

For every $\epsilon>0$ there is $R_{0}>0$ such that for each $R \geq R_{0}$ we have

$$
\inf _{S>R} \frac{\omega(S)}{\log \left(\frac{S}{R}\right)} \leq \epsilon \frac{\sigma(R)}{\log \left(\frac{R}{\sigma(R)}\right)} .
$$

The limit case $t=1$, which occurs when we replace the Roumieu class $\mathcal{E}_{\{\sigma\}}\left(\mathbb{R}^{N}\right)$ by the space of real-analytic functions, is the content of corollary 18 .

Ehrenpreis [7, Theorem II, p. 556] proved that, in case $\omega=\log$, the intersection of the ranges of all the convolution operators on $\mathcal{E}_{(\omega)}\left(\mathbb{R}^{N}\right)$ coincides with the space of the real-analytic functions, showing that the phenomenon in the example cannot happen in the larger class of arbitrary convolution operators.

Proposition 17. Let $\mu \in \mathcal{E}_{(\omega)}^{\prime}\left(\mathbb{R}^{N}\right)$. The following conditions are equivalent:

(a) The space $A\left(\mathbb{R}^{N}\right)$ of real analytic functions is contained in $T_{\mu}\left(\mathcal{E}_{(\omega)}\left(\mathbb{R}^{N}\right)\right)$. 
(b) $\mu$ satisfies the following condition $(E)$ :

For each $m \in \mathbb{N}$ there exists $R>0$ such that for each $x \in \mathbb{R}^{N}$ with $|x| \geq R$ there exists $\xi \in \mathbb{C}^{N}$ satisfying $|x-\xi| \leq \frac{1}{m}|x|$ such that $|\hat{\mu}(\xi)| \geq \exp \left(-\frac{|\xi|}{m}\right)$.

(c) There exists a weight function $\sigma, \sigma \geq \omega$, such that $T_{\mu}: \mathcal{E}_{(\sigma)}\left(\mathbb{R}^{N}\right) \rightarrow \mathcal{E}_{(\sigma)}\left(\mathbb{R}^{N}\right)$ is surjective.

Proof. (a) $\Rightarrow$ (b). We proceed by contradiction. We assume that there are $k \in \mathbb{N}$ and an unbounded sequence $\left(x_{j}\right)$ in $\mathbb{R}^{N}$ such that for each $j \in \mathbb{N}$ and each $\xi \in \mathbb{C}^{N}$ with $\left|\xi-x_{j}\right| \leq \frac{\left|x_{j}\right|}{k}$ we have $|\hat{\mu}(\xi)| \leq \exp \left(-\frac{|\xi|}{k}\right)$. Let $\left(f_{j}\right)$ the sequence of entire functions given by lemma 1 with $v(t):=t$ and $R_{j}:=\frac{\left|x_{j}\right|}{3 k}$.

For $\xi \in \mathbb{C}^{N}$ with $\left|\xi-x_{j}\right| \geq R_{j}+2$ we have

$$
\left|f_{j}(\xi)\right| \leq C_{N} \exp \left(|\operatorname{Im} \xi|+2+C_{N} \log \left(1+|\xi|^{2}\right)\right),
$$

which implies $f_{j}$ is the Fourier-Laplace transform of some distribution with compact support. Let $\nu_{j} \in \mathcal{E}_{(\omega)}^{\prime}\left(\mathbb{R}^{N}\right)$ with $\hat{\nu}_{j}=f_{j}$. We show that $\left\{T_{\mu}^{t}\left(\nu_{j}\right): j \in \mathbb{N}\right\}$ is a bounded set in $\mathcal{E}_{(\omega)}^{\prime}\left(\mathbb{R}^{N}\right)$. If $\left|\xi-x_{j}\right|<R_{j}+2$, then

$$
\left|f_{j}(\xi)\right| \leq C_{N} \exp \left(R_{j}+4+C_{N} \log \left(1+|\xi|^{2}\right)\right) \text {. }
$$

and

$$
\left|\widehat{\tilde{\mu}}(\xi) f_{j}(\xi)\right| \leq C_{N} \exp \left(\frac{-3|\xi|+\left|x_{j}\right|}{3 k}+4+C_{N} \log \left(1+|\xi|^{2}\right)\right) .
$$

Since $\left|x_{j}\right|<|\xi|+R_{j}+2=|\xi|+\frac{\left|x_{j}\right|}{3 k}+2$, we have $\left|x_{j}\right| \leq 3|\xi|$ for $j$ large enough and

$$
\left|\widehat{\widetilde{\mu}}(\xi) f_{j}(\xi)\right| \leq C_{N} \exp \left(4+C_{N} \log \left(1+|\xi|^{2}\right)\right)
$$

for every $\xi \in \mathbb{C}^{N}$ with $\left|\xi-x_{j}\right|<R_{j}+2, j \geq j_{0}$. Moreover, $\left|\xi-x_{j}\right| \geq R_{j}+2$ implies

$$
\left|\widehat{\tilde{\mu}}(\xi) f_{j}(\xi)\right| \leq|\widehat{\tilde{\mu}}(\xi)| C_{N} \exp \left(|\operatorname{Im} \xi|+2+C_{N} \log \left(1+|\xi|^{2}\right)\right),
$$

whence it follows that $\left\{T_{\mu}^{t}\left(\nu_{j}\right): j \in \mathbb{N}\right\}$ is a bounded set in $\mathcal{E}_{(\omega)}^{\prime}\left(\mathbb{R}^{N}\right)$. We can proceed as in the lemma 11 to conclude that $\left\{\nu_{j} \mid A\left(\mathbb{R}^{N}\right): j \in \mathbb{N}\right\}$ is equicontinuous in $A^{\prime}\left(\mathbb{R}^{N}\right)$, i.e. $([11,15.1 .5])$

$$
\exists A>0 \quad \forall \epsilon>0: \sup _{j} \sup _{z}\left|f_{j}(z)\right| \exp (-A|\operatorname{Im} z|-\epsilon|z|)<\infty .
$$

On the other hand,

$$
\left|f_{j}\left(x_{j}\right)\right| \exp \left(-\epsilon\left|x_{j}\right|\right) \geq \exp \left(\left(\frac{2}{3 k \pi \sqrt{N}}-\epsilon\right)\left|x_{j}\right|-C_{N} \log \left(1+\left|x_{j}\right|^{2}\right)\right) .
$$

Now, for $0<\epsilon<\frac{2}{3 k \pi \sqrt{N}}$, this implies $\lim _{j \rightarrow \infty}\left|f_{j}\left(x_{j}\right)\right| \exp \left(-\epsilon\left|x_{j}\right|\right)=\infty$, which is a contradiction.

(b) $\Rightarrow$ (c). Applying (b) inductively, we find a strictly increasing sequence $\left(R_{m}\right)$ tending to infinity so that the conclusion of (b) holds with $m$ replaced by $2 m$ for all $x \in \mathbb{R}^{N}$ satisfying $|x| \geq R_{m}$. Then we define $g(t):=0$ for $t \in\left[0, R_{1}\left[\right.\right.$ and $g(t):=\frac{t}{m}$ for $t \in\left[R_{m}, R_{m+1}[\right.$. Since $g(t)=o(t)$ as $t$ goes to infinity we can apply [6] $1.7 ; 1.8$ ] to get a weight function $\sigma \geq \omega$ such that $g(t)=o(\sigma(t))$ and $\sigma(t)=o(t)$ as $t$ goes to infinity. Proceeding as in [5, 3.2] we conclude that $\mu$ is slowly decreasing for $(\sigma)$. It follows from corollary 6 that $T_{\mu}: \mathcal{E}_{(\sigma)}\left(\mathbb{R}^{N}\right) \rightarrow \mathcal{E}_{(\sigma)}\left(\mathbb{R}^{N}\right)$ is surjective. 
We now include some result on the limit case of the space of real analytic functions. Compare with example 13.

Corollary 18. Let $\omega$ be an arbitrary weight and let $\mu \in \mathcal{E}_{(\omega)}^{\prime}\left(\mathbb{R}^{N}\right)$ satisfy $\operatorname{supp} \mu=$ (0). Then the range of $T_{\mu}: \mathcal{E}_{(\omega)}\left(\mathbb{R}^{N}\right) \longrightarrow \mathcal{E}_{(\omega)}\left(\mathbb{R}^{N}\right)$ contains the space $A\left(\mathbb{R}^{N}\right)$ of real analytic functions.

Proof. In fact, $\mu$ satisfies the condition (E) by [17, Th.7; Lemme 15] (see also [14. $1.8])$.

The next result was already proved in [4] with a different approach. It should be compared with corollary 18 and example 16 where a restriction on the support of $\mu$ was needed.

Corollary 19. Let $\omega$ be a non-quasianalytic weight and let $\mu \in \mathcal{E}_{(\omega)}^{\prime}\left(\mathbb{R}^{N}\right)$. Then the space $A\left(\mathbb{R}^{N}\right)$ of real analytic functions is contained in the range of the convolution operator $T_{\mu}: \mathcal{E}_{(\omega)}\left(\mathbb{R}^{N}\right) \longrightarrow \mathcal{E}_{(\omega)}\left(\mathbb{R}^{N}\right)$.

Proof. It follows from [7, 4.5], after taking convolution with a fixed test function, that $\mu$ satisfies condition (E).

Lemma 20. Let $\mu \in A^{\prime}\left(\mathbb{R}^{N}\right)$ be an analytic functional. Then there are a (quasianalytic) weight $\sigma$ and an ultradistribution $\nu \in \mathcal{E}_{(\sigma)}^{\prime}\left(\mathbb{R}^{N}\right)$ such that $\mu$ is the restriction of $\nu$ to $A\left(\mathbb{R}^{N}\right)$.

Proof. In fact, for every $\epsilon>0$ we put

$$
C_{\epsilon}:=\sup _{z \in \mathbb{C}}|\hat{\mu}(z)| \exp (-|\operatorname{Im} z|-\epsilon|z|)<\infty
$$

and define $g:\left[0,+\infty\left[\rightarrow\left[0,+\infty\left[\right.\right.\right.\right.$ by $\exp (g(t)):=\sup _{|z|=t}|\hat{\mu}(z)| \exp (-|\operatorname{Im} z|)$. Then $\exp (g(t)) \leq C_{\epsilon} \exp (\epsilon t)$, from where it follows that $g(t)=o(t)$ as $t$ goes to $\infty$. Now we apply [6, 1.7,1.8] to find a weight $\sigma$ such that $g(t)=o(\sigma(t))$ and $\sigma(t)=o(t)$. Then, $g(|z|) \leq K \sigma(|z|)$ for some $K>0$ and every $z \in \mathbb{C}$ and

$$
\sup _{z \in \mathbb{C}}|\hat{\mu}(z)| \exp (-|\operatorname{Im} z|-K \sigma(|z|))<\infty .
$$

If $p$ is a subharmonic function on $\mathbb{C}$ satisfying a growth condition of the form $p(z) \leq A+B|z|, z \in \mathbb{C}$, then the limit set $L(p)$ of $p$ is the set of all subharmonic functions in $\mathbb{C}$ which are limits in $L_{\text {loc }}^{1}\left(\mathbb{R}^{2}\right)$ (or equivalently in $\mathcal{D}^{\prime}\left(\mathbb{R}^{2}\right)$ ) of sequences of the form $t_{j}^{-1} p\left(t_{j}.\right)$, where $t_{j}$ goes to infinity. The indicator function $j_{p}$ of $p$ is the least upper semicontinuous majorant of $\varlimsup_{t \rightarrow \infty} t^{-1} p(t$.). The indicator function $i_{f}$ of an entire function $f$ is the indicator function of the subharmonic function $p=\log |f|$. The next theorem depends heavily on a result of Sigurdsson [30] on the existence of analytic functions $f$ such that $\log |f|$ has the largest limit set among all subharmonic functions having a fixed subharmonic and positively homogeneous function $r$ as indicator function.

Theorem 21. There are a quasianalytic weight $\sigma$ and an ultradistribution $\mu \in$ $\mathcal{E}_{(\sigma)}^{\prime}(\mathbb{R})$ such that the range of $T_{\mu}: \mathcal{E}_{(\sigma)}(\mathbb{R}) \longrightarrow \mathcal{E}_{(\sigma)}(\mathbb{R})$ does not contain the space $A(\mathbb{R})$ of real analytic functions.

Proof. We take $r(z):=|\operatorname{Im} z|$ and apply [30, 1.3.2] to get an entire function $f \in$ $H(\mathbb{C})$ such that the limit set of $p:=\log |f|$ coincides with

$$
L(p)=\{u \text { subharmonic : } u(0)=0, u(z) \leq|\operatorname{Im} z|\} .
$$


By [30, 1.1.2] (see also [31, 2.2(ii)]), the indicator function of $f$ is given by $i_{f}(z):=$ $\varlimsup_{t \rightarrow \infty} \frac{p(t z)}{t}=|\operatorname{Im} z|, z \in \mathbb{C}$. From the Hartogs lemma [16] it follows that for all $\epsilon>0$ there is $t_{\epsilon}$ such that $\frac{p(t z)}{t} \leq|\operatorname{Im} z|+\epsilon$ for every $t \geq t_{\epsilon},|z|=1$. Consequently $f$ is the Fourier-Laplace transform of an analytic functional. We apply the lemma 20 to find a quasianalytic weight $\sigma$ and an ultradistribution $\mu \in \mathcal{E}_{(\sigma)}^{\prime}(\mathbb{R})$ such that $f=\hat{\mu}$. To finish we show that $f$ does not satisfy the condition (E). To do this, we put $u(z):=\log |\cos z|$, which is an element of $L(p)$. There is an increasing and unbounded sequence $\left(t_{j}\right)$ such that $\lim _{j \rightarrow \infty} t_{j}^{-1} \log \left|f\left(t_{j}.\right)\right|=u($.$) in L_{\mathrm{loc}}^{1}\left(\mathbb{R}^{2}\right)$. We take $x_{0}=\frac{\pi}{4}$ and $0<\epsilon<1$ satisfying $u(z)<-2 \epsilon$ for every $z \in \mathbb{C}$ with $\left|z-x_{0}\right| \leq \epsilon$. Since

$$
\varlimsup_{j \rightarrow \infty}\left(\sup _{\left|z-x_{0}\right| \leq \epsilon} t_{j}^{-1} \log \left|f\left(t_{j} z\right)\right|\right) \leq \sup _{\left|z-x_{0}\right| \leq \epsilon} u(z) \leq-2 \epsilon
$$

by [10, 4.1.9], there is $j_{0} \in \mathbb{N}$ such that $t_{j}^{-1} \log \left|f\left(t_{j} z\right)\right| \leq-2 \epsilon$ for every $j \geq j_{0}$ and $\left|z-x_{0}\right| \leq \epsilon$. Then, as $|z| \leq\left|x_{0}\right|+1$, we obtain $\log \left|f\left(t_{j} z\right)\right| \leq-2 \epsilon^{\prime}\left|t_{j} z\right|$ for every $j \geq j_{0}$ and $\left|z-x_{0}\right| \leq \epsilon$, with $\epsilon^{\prime}=\frac{\epsilon}{\left|x_{0}\right|+1}$. Finally we put $a_{j}:=t_{j} x_{0}, j \geq j_{0}$. Then $|f(\xi)| \leq \exp \left(-2 \epsilon^{\prime}|\xi|\right)$ for every $\xi \in \mathbb{C}$ with $\left|\xi-a_{j}\right| \leq \epsilon^{\prime}\left|a_{j}\right|$ and $f$ does not satisfy condition $(\mathrm{E})$.

By Sigurdsson [31, Thm. and Def. 2.5], for each $\mu \in \mathcal{E}_{(\sigma)}^{\prime}\left(\mathbb{R}^{N}\right), \hat{\mu}$ satisfies condition (E) if and only if $u(x)=0$ for all $x \in \mathbb{R}^{N}$ and $u \in L(\log |\hat{\mu}|)$. (The uniformity of $R$-which a priori depends on the direction $x \in \mathbb{R}^{N} \backslash(0)$-follows via [31, 2.5 (v)] from Hartogs' lemma [16, Thm. 1.31]. See also [16 Thm. 4.9].) These conditions are equivalent to several notions of regular growth of $|\hat{\mu}|$ on the cone $\mathbb{R}^{N}([31,2.5])$. By Hörmander and Sigurdsson [12, in the case $N=1$, the equivalent conditions on $\hat{\mu}$ are fulfilled if $\omega$ is a non-quasianalytic weight. Of course, the function $f$ in Theorem 21 is not of regular growth on $\mathbb{R}$.

\section{REFERENCES}

1. V.S. Azarin, On the asymptotic behaviour of subharmonic functions of finite order. Math. USSR Sb. 36 (1980), 134-154. English transl. MR 81e:31001

2. C.A. Berenstein, B.A. Taylor, Interpolation problems in $\mathbb{C}^{N}$ with applications to harmonic analysis. J. Analyse Math 38 (1980), 188-254. MR 82h:32002

3. - A new look at interpolation theory for entire functions of one variable. Adv. in Math. 33 (1979), 109-143. MR 80j:30023

4. J. Bonet, A. Galbis, The range of non-surjective convolution operators on Beurling spaces. Glasgow Math. J. 38 (1996), 125-135. MR 97g:46032

5. J. Bonet, A. Galbis, R. Meise, On the range of convolution operators on non-quasianalytic ultradifferentiable functions. Studia Math. 126 (2) (1997), 171-198. MR 99a:46071

6. R. Braun, R. Meise, B.A. Taylor, Ultradifferentiable functions and Fourier Analysis. Resultate Math. 17 (1990), 206-237. MR 91h:46072

7. L. Ehrenpreis, Solution of some problems of division, Part IV. Invertible and elliptic operators. Amer. J. Math. 82 (1960), 522-588. MR 22:9848

8. L. Hörmander, On the range of convolution operators. Ann. of Math. 76 (1962), 148-170. MR 25:5379

9. - An Introduction to Complex Analysis in Several Variables. Van Nostrand, Princeton, N.J., 1966; (3rd ed.) North-Holland, Amsterdam, 1990. MR 91a:32001 
10. — The analysis of linear partial differential operators I. Springer, 1983. MR 85g:3500a

11. The analysis of linear partial differential operators II. Springer Verlag, 1983. MR 85g:3500b

12. L. Hörmander, R. Sigurdssson, Growth properties of plurisubharmonic functions related to Fourier-Laplace transforms. Lund University, 1993.

13. J.J. Kelleher, B.A. Taylor, Closed ideals in locally convex algebras J. Reine Angew. Math. 255 (1972), 190-209. MR 46:6046

14. M. Langenbruch, Continuous linear right inverses for convolution operators in spaces of real analytic functions. Studia Math. 110 (1) (1994), 65-82. MR 95f:46061

15. B.J. Levin, Distribution of zeros of entire functions. AMS, Providence, Rhode Island 1980. MR 81k:30011

16. P. Lelong, L. Gruman, Entire functions of several complex variables. Springer Verlag, 1986. MR 87j:32001

17. A. Martineau, Équations différentielles d'ordre infini. Bull. Soc. Math. France 95 (1967), 109-154.

18. R. Meise, B.A. Taylor, D. Vogt, Equivalence of slowly decreasing conditions and local Fourier expansion. Indiana Univ. Math. J. 36 (1987), 729-756. MR 89c:46058

19. — Continuous linear right inverses for partial differential operators on nonquasianalytic classes and on ultradistributions. Math. Nachr. 180 (1996), 213-242. MR 97j:46036

20. R. Meise, D. Vogt, Introduction to Functional Analysis. Clarendon Press, Oxford, 1997. MR 98g:46001

21. T. Meyer, Die Fourier-Laplace-Transformation quasianalytischer Funktionale und ihre Anwendung auf Faltungsoperatoren. Diplomarbeit, Düsseldorf, 1989.

22. - Surjectivity of convolution operators on spaces of ultradifferentiable functions of Roumieu type. Studia Math. 125 (1997) 101-129. MR 98e:46045

23. S. Momm, Closed principal ideals in nonradial Hörmander algebras. Archiv Math. 58 (1992), 47-55. MR 93a:46106

24. Division problems in spaces of entire functions of finite order. Functional Analysis (Ed. K.D. Bierstedt, A. Pietsch, W. Ruess, D. Vogt) (Marcel Dekker, 1993), 435-457. MR 95a:46035

25. A A Phragmén-Lindelöf theorem for plurisubharmonic functions on cones in $\mathbb{C}^{N}$. Indiana Univ. Math. J. 41 (1992) 861-867. MR 93j:32023

26. - A division problem in the space of entire functions of exponential type. Ark. Mat. 32 (1994) 213-236. MR 95b:32003

27. T. Rösner, Surjektivität partieller Differentialoperatoren auf quasianalytischen RoumieuKlassen. Dissertation. Düsseldorf 1997.

28. L.A. Rubel, B.A. Taylor, A Fourier series method for meromorphic and entire functions. Bull. Soc. Math. France 96 (1968), 53-96. MR 42:509

29. L.A. Rubel, Entire and meromorphic functions. Springer-Verlag, New York, 1996. MR 97c:30001

30. R. Sigurdsson, Growth properties of analytic and plurisubharmonic functions of finite order. Math. Scand. 59 (1986), 235-304. MR 88m:32002

31. Convolution equations in domains of $\mathbb{C}^{N}$. Ark. Mat. 29 (1991), 285-305. MR 93b:46074

32. D. Vogt, Topics on projective spectra of (LB)-spaces. In Advances in the theory of Fréchet spaces, T. Terzioglu (Ed.), Istanbul 1987, NATO ASI Series C, Vol. 287, Kluwer, Dordrecht, 1989, 11-27. MR 93b:46011

Departamento de Matemática Aplicada, E.T.S. Arquitectura, E-46071 Valencia, Spain E-mail address: jbonet@mat.upv.es

Departamento de Análisis Matemático, Universidad de Valencia, E-46100 Burjasot (VALENCIA), SPAin

E-mail address: Antonio.Galbis@uv.es

Mathematisches Institut, Heinrich-Heine-Universität, D-40225 Düsseldorf 1, GerMANY

E-mail address: momm@mx.cs.uni-duesseldorf.de, siegfried.momm@t-online.de 